\title{
Praxis der antithrombotischen Therapie vor grundlegendem Wandel
}

\author{
Bei antikoagulierten Patienten, die einen Koronarstent brauchen, reichen Antikoagulans und Clo- \\ pidogrel aus, um Thrombosen in Schach zu halten. Mehr noch: Acetylsalicylsäure (ASS) wegzu- \\ lassen reduziert neuen Daten zufolge signifikant das Blutungsrisiko ebenso wie die Sterberate.
}

„Das ist ein Blockbuster-Vortrag, der die klinische Praxis über Nacht und die Leitlinien bei nächster Gelegenheit verändern wird“, kommentierte kein geringerer als der Präsident des American College of Cardiology, Professor David Holmes von der Mayo Clinic in Rochester, die Ergebnisse der beim ESC-Kongress vorgestellten WOEST-Studie.

Es geht um ein häufiges klinisches Problem: Ein Patient, der etwa wegen Vorhofflimmern oder mechanischen Herzklappen oral antikoaguliert werden muss, erkrankt zusätzlich an einer koronaren Herzkrankheit und wird mit einem Koronarstent versorgt. Nun benötigt er laut Leitlinie zusätzlich zwei Thrombozytenhemmern nämlich ASS plus Clopidogrel. Doch bei einer Kombination von insgesamt drei antithrombotischen Wirksubstanzen steigt das Blutungsrisiko an. Das Dilemma: Bei Verzicht auf orale Antikoagulation droht ein Schlaganfall, bei Reduktion der Thrombozytenhemmung eventuell eine Stentthrombose. In der randomisierten niederländisch/belgischen WOEST-Studie ist erstmals bei 573 antikoagulierten $\mathrm{Pa}$ tienten, die aufgrund einer KHK eine Koronarintervention mit Stent erhielten, geprüft worden, ob bei diesen Patienten auf ASS verzichtet werden kann. Die Hälfte der Patienten erhielt Antikoagulation plus Clopidogrel, die andere Hälfte zusätzlich ASS. So behandelt wurde ein Jahr nach Implantation eines beschichteten Stents (DES, 2/3 der Patienten), oder mindestens ein Monat nach Positionierung eines unbeschichteten Stents (BMS, 1/3 der Patienten). Bei 25\% der Patienten lag ein akutes Koronarsyndrom (ACS) vor. Das Follow-up betrug ein Jahr. Die Autoren um Professor Willem Dewilde, Tweesteden Hospital in Tilburg/NL, erhofften sich, dass bei Verzicht auf ASS das Blutungsrisiko sinkt, ohne dass die thrombotischen Komplikationen ansteigen. Doch die Ergebnisse übertrafen die Erwartungen, denn die Patienten hatten sogar einen Mortalitätsvorteil: 2,6\% vs. $6,4 \%$ der Patienten waren nach 1 Jahr gestorben $(\mathrm{p}=0,027)$.

\section{Blutungsrisiko halbiert und ein Trend zu weniger thrombotischen Komplikationen}

Blutungen (primärer Endpunkt) waren nach 1 Jahr mit 19,5\% vs. $44,9 \%$ ebenfalls signifikant seltener aufgetreten, wenn ASS weggelassen wurde. Den Unterschied machten aber überwiegend leichtere Blutungen der Haut und des Gastrointestinaltraktes aus. Schwere Blutungen waren nur im Trend seltener (3,3\% vs. 5,8\%). In beiden Gruppen gab es 3 ei intrakranielle Blutungen.

Der Sicherheitsvorteil musste nicht mit einem Wirksamkeitsverlust erkauft werden, im Gegenteil: Überraschenderweise waren Herzinfarkte (3,3\% vs. $4,7 \%)$, Schlaganfälle (1,1\% vs. $2,9 \%)$

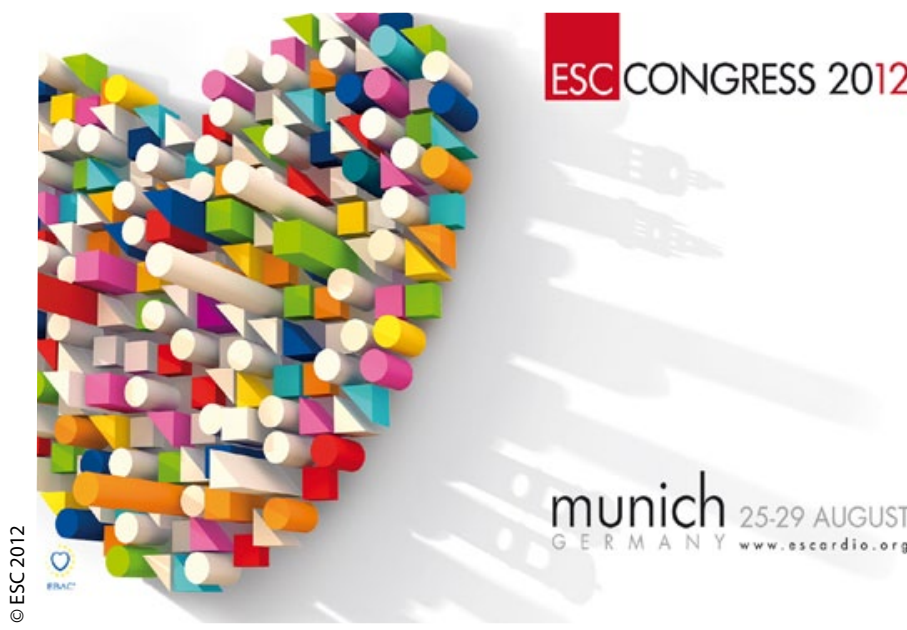

und Stent-Thrombosen (1,5\% vs. 3,2\%) unter dem 2-Medikamenten-Regime seltener als unter dem 3-Medikamenten-Regime. Der kombinierte sekundäre Endpunkt (Tod, Infarkt, Schlaganfall, Stentthrombose, Revaskularisierung) war in Summe mit $11,3 \%$ unter dualer Therapie signifikant seltener $(\mathrm{p}=0,025)$ als unter der Triple-Therapie mit 17,7\%. Allerdings war die Studie zu klein, um statistisch eine Nicht-Unterlegenheit bei diesem Endpunkt zu beweisen.

Die Frage ist nun: Können diese Daten auch extrapoliert werden auf Patienten, die statt Clopidogrel Ticagrelor oder Prasugrel erhalten, oder auf Patienten, die statt Vitamin-K-Antagonisten Dabigatran oder Rivaroxaban erhalten? Aufgrund der fehlenden Daten wäre das ein wenig zu weit extrapoliert, warnte Holmes.

Dr. med. Dirk Einecke

Quelle:Jahrestagung European Society of Cardiology (ESC) vom 25.29.8.2012 in München. Session Late Breaking Trials on Prevention to Heart Failure (Hotline 2) .

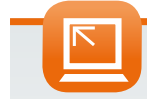

Mehr Informationen auf springermedizin.de

Dossier „ESC-Kongress"

Beiträge zum ESC-Kongress 2012 in

München unter:

www.springermedizin.de/esc-2012 